\title{
Fetal Bradyarrhythmia
}

National Cancer Institute

\section{Source}

National Cancer Institute. Fetal Bradyarrhythmia. NCI Thesaurus. Code C111770.

An abnormality in the fetal heart rhythm associated with a decrease in fetal heart rate below 110 beats per minute. 